\title{
Dépistage du carcinome du côlon: remboursement par l'assurance de base
}

\author{
Le dépistage du carcinome du côlon est couvert depuis juillet 2013 par l'assurance \\ obligatoire des soins pour toutes les personnes de 50 à 69 ans. Le Département \\ fédéral de l'intérieur a ainsi partiellement répondu à la requête déposée par la Ligue \\ suisse contre le cancer. Celle-ci réclame néanmoins toujours la mise en place d'un \\ dépistage organisé.
}

\section{Cornelia Schwager}

Collaboratrice spécialisée Prévention et dépistage, Ligue suisse contre le cancer

\section{Un cancer fréquent}

Souvent diagnostiqué tardivement, le carcinome du côlon est, avec 4100 nouveaux cas par an environ, l'un des cancers les plus fréquents en Suisse. Or, dépisté à un stade peu avancé, il offre de bonnes chances de guérison.

\section{Pour soutenir les professionnels dans leur travail de conseil la Ligue contre le cancer a élaboré divers documents d'information.}

\section{Références \\ 1 Ordonnance sur les prestations de l'assurance des soins (OPAS, RS 832.112.31); art. $12 \mathrm{~d}$, lettre b: Mesures en vue du dépistage précoce de maladies chez certains groupes à risques. \\ 2 Demande de prise en charge par l'assurance obligatoire des soins des coûts des prestations liées à un programme de dépistage du côlon, déposée par la Ligue suisse contre le cancer avec le collectif responsable du PDCC (2011, 2012, 2013). \\ 3 Ordonnance sur les prestations de l'assurance des soins (OPAS, RS 832.112.31); art. 12e, lettre d: Mesures de dépistage précoce de maladies dans toute la population.}

\section{Correspondance: \\ Cornelia Schwager \\ Ligue suisse contre le cancer \\ Effingerstrasse 40 \\ CH-3001 Berne \\ Tél. 0313899358}

\section{cornelia.schwager[at]}

liguecancer.ch

\section{Une requête motivée par l'absence de base légale}

Jusqu'à il y a peu, le dépistage du carcinome du côlon n'était remboursé que pour des groupes à risque strictement définis [1]. En 2011, la Ligue suisse contre le cancer a, avec d'autres organisations, soumis au Département fédéral de l'intérieur (DFI) une demande de prise en charge par l'assurance obligatoire des soins des coûts des prestations liées à un programme de dépistage du carcinome du côlon (PDCC) [2]. Le PDCC a pour objectif d'augmenter la détection précoce du carcinome du côlon et de contribuer ainsi à abaisser la mortalité. Il vise parallèlement à garantir l'assurance-qualité et l'égalité des chances. La requête est soutenue par le Collège de médecine de premier recours, la Société suisse de gastroentérologie, la Société suisse de chirurgie viscérale, la Ligue gastrointestinale Suisse, pharmaSuisse et swiss cancer screening.

\section{Un pas dans la bonne direction}

Depuis le $1^{\text {er }}$ juillet 2013, le dépistage du carcinome du côlon figure dans le catalogue des prestations à charge de l'assurance obligatoire des soins pour les personnes de 50 à 69 ans. Sont couverts un test de recherche de sang occulte dans les selles (selon la liste des analyses) tous les deux ans ainsi que la coloscopie subséquente en cas de test positif ou une coloscopie tous les dix ans [3]. Cette prestation est remboursée sans qu'elle s'inscrive dans un programme et sans suppression de la franchise (contrairement au dépistage systématique du cancer du sein). Si un canton souhaite introduire un programme de dépistage, le DFI étudiera néanmoins la possibilité de libérer l'examen de la franchise. Les programmes pilotes cantonaux revêtent une importance primordiale; ils permettent en effet de clarifier des questions liées au dépistage systématique du carcinome du côlon pour l'ensemble du pays. Il est donc indispensable de les soutenir et de les encourager.

La Ligue suisse contre le cancer et le collectif responsable du PDCC considèrent la décision du DFI en faveur d'un meilleur dépistage du carcinome du côlon comme un pas dans la bonne direction. Ils n'en réclament pas moins toujours la mise en place d'un dépistage organisé.

\section{Informer les professionnels et la population}

L'introduction d'un dépistage systématique du carcinome du côlon à l'échelle du pays ne se fera sans doute pas avant plusieurs années. D'ici là, les médecins assument un rôle d'autant plus important en tant que personnes de confiance: c'est à eux qu'il appartient d'informer leurs patients de la possibilité d'effectuer un dépistage et de les conseiller et soutenir dans le choix de la méthode. Il est essentiel d'informer avec soin les hommes et les femmes de la tranche d'âge visée pour qu'ils puissent prendre leur décision et profiter d'une qualité aussi élevée que possible pour l'examen.

Pour soutenir les professionnels dans leur travail de conseil et aider les hommes et les femmes à prendre leur décision, la Ligue contre le cancer a élaboré divers documents d'information. Ceux-ci seront disponibles à la page www.liguecancer.ch/cancerintestin dès le mois de mars, mois traditionnellement dédié au cancer de l'intestin dans le monde entier. 